\title{
ANÁLISE DA DISTRIBUIÇÃO GEOGRÁFICA DE COVID-19 NA MESORREGIÃO SUL/SUDOESTE DE MINAS GERAIS
}

\section{ANALYSIS OF THE GEOGRAPHICAL DISTRIBUTION OF COVID-19 IN THE SOUTH/SOUTHWESTERN MESOREGION OF MINAS GERAIS}

\author{
Sérgio Henrique de Oliveira Teixeira \\ Doutor em Geografia UNICAMP \\ IFSULDEMINAS \\ sergio.teixeira@ifsuldeminas.edu.br \\ André Lopes de Souza \\ Mestrando em Geografia UERJ \\ Instituto Brasileiro de Geografia e Estatística \\ souzalandre1@gmail.com
}

\begin{abstract}
RESUMO
A pandemia de COVID-19 apresenta desafios significativos para o Brasil. O conhecimento geográfico pode contribuir para compreender a dinâmica de distribuição dessa doença e assim compor mecanismos de seu enfrentamento. A partir do recorte da mesorregião sul/sudoeste de Minas Gerais, analisou-se como o novo coronavírus se disseminou nesses municípios, quais os principais nexos geográficos e os riscos. Para isso, analisou-se geograficamente um conjunto de informações como hierarquia urbana, população total e proporção de idosos, casos confirmados e mortes em decorrência da doença, e o papel das vias de transporte. Como conclusão, aponta-se para o destacado papel da estruturação da rede urbana e dos eixos rodoviários na disseminação da COVID-19 no sul de Minas Gerais. Elabora-se também a hipótese a ser verificada por pesquisas posteriores de que os estabelecimentos que margeiam as rodovias (postos de gasolina, conveniência, restaurantes, borracheiros, etc) sejam eixos de disseminação da doença. É proposto ao poder público a manutenção do isolamento social e a instalação de postos de controle nas entradas rodoviárias dos municípios, visando a medição de temperatura e orientação dos cidadãos.
\end{abstract}

Palavras-chave: COVID-19. Sul de Minas Gerais. geografia médica e da saúde.

\begin{abstract}
The COVID-19 pandemic presents significant challenges for Brazil. Geographic knowledge can contribute to understand the distribution dynamics of this disease and thus compose mechanisms for its coping. From the geography of the south / southwest mesoregion of Minas Gerais, it was analyzed how the new coronavirus spread in these municipalities, which are the main geographical links and the risks. For this, a set of information was geographically analyzed, such as urban hierarchy, total population and proportion of elderly people, confirmed cases and deaths due to the disease, and the role of transport routes. As a conclusion, we point out the prominent role of structuring the urban network and the road axes in the dissemination of COVID-19 in the south of Minas Gerais. It is also elaborated the hypothesis to be verified by subsequent researches that the establishments that border the highways (gas stations, convenience stores, restaurants, tire repairers, etc.) are keys for the spread of the disease. It is proposed to the public authorities to maintain social isolation and to install checkpoints at the road entrances of the municipalities, aiming at the measurement of temperature and orientation of citizens.
\end{abstract}

Keywords: COVID-19. South of Minas Gerais. Medical and health geography.

Recebido em: 10/05/2020

Aceito para publicação em: 21/05/2020. 


\section{INTRODUÇÃO}

Este artigo busca organizar um levantamento de dados sobre a pandemia causada pela disseminação da COVID-19 de modo a oferecer informações e diagnósticos atuais e precisos da transmissão para conhecimento e análise pública. Em especial, interessa fornecer um mapeamento e uma organização dos dados que possam auxiliar os tomadores de decisão das cidades localizadas na mesorregião Sul e Sudoeste de Minas Gerais.

Neste momento de grandes desafios para o Brasil, a Geografia contribui para elucidar a dimensão espacial da pandemia. Pode-se dizer que "a Geografia é o campo de estudos que interpreta as razões pelas quais coisas diversas estão situadas em posições diferentes ou por que as situações espaciais diversas podem explicar qualidades diferentes de objetos, coisas, pessoas e fenômenos" (GOMES, 2017, p. 20). No caso da COVID-19, a Geografia é um dos elementos explicativos sobre como os municípios são afetados diferentemente pela doença. Portanto, é um saber necessário para a elaboração de estratégias de enfrentamento da pandemia em diversas escalas.

As informações com relação à pandemia são inúmeras e, portanto, a análise de forma precisa e com melhor qualidade necessita de um grande arcabouço de técnicas e investigações. A mídia frequentemente recorre a mapas para a visualização da evolução da pandemia, o que facilita o entendimento do público. Entretanto, um olhar geográfico rigoroso traz novas perspectivas sobre a COVID-19, colocando-se em questão outros debates e problemas. Não se desqualifica o olhar midiático, que cumpre função essencial de comunicação com o público. Trata-se de entender de maneira precisa e científica a dinâmica geográfica de distribuição do vírus e as formas de seu enfrentamento.

Propomos, ao longo do trabalho, uma breve introdução sobre a questão da difusão de doenças na teoria geográfica, em seguida uma discussão sobre a distribuição da doença na região delimitada e, por fim, uma indicação dos desafios que a sociedade e os governos terão durante e após a pandemia, do ponto de vista geográfico.

\section{POR UMA GEOGRAFIA DOS FLUXOS: O CASO DA COVID-19}

A ascensão do Meio Técnico Científico Informacional - MTCI - (SANTOS, 1996) a partir do pósguerra, aponta uma maior espessura dos fluxos na organização da sociedade e dos territórios. É a partir do período pós-guerra que a circulação passa a se realizar em mais alta velocidade por meio de sua articulação com os sistemas informacionais ${ }^{2}$. Catão (2011), aponta que as inúmeras mudanças socioespaciais decorrentes da ascensão do $\mathrm{MTCl}$ - como o avanço das técnicas de comunicação e de transporte - somadas a destruição ocasionada no sudeste asiático no pós-guerra, propiciaram a emergência de doenças virais como um problema de saúde pública global, assumindo também formas mais graves e letais. Segundo o autor, ao analisar os estudos de Gubler (1998), para o caso da Dengue, esse novo padrão coaduna para que grandes epidemias ocorram com maior frequência, acometendo um número grande de pessoas. As transformações territoriais que contribuíram para tais mudanças, em especial para os países de capitalismo dependente (MARINI,1977), podem ser classificadas segundo as mudanças do MTCl. Estão entre elas, segundo Santos (2005; 2011):

- Aumento da população mundial;

- Adensamento de áreas urbanas;

- Degradação as Infraestruturas básicas;

- Aumento das Aglomerações Industriais;

- Acúmulo de resíduos de todo ordem sem tratamento adequado;

- Intenso aumento dos fluxos no território (materiais e imateriais);

- Diminuição da seguridade social das populações mais pobres, em especial a desassistência em saúde pública;

- Agravamento de condições sanitárias precárias da maioria da população.

2 "Neste período, os objetos técnicos tendem a ser ao mesmo tempo técnicos e informacionais, já que, graças à extrema intencionalidade de sua produção e de sua localização, eles já surgem como informação; e, na verdade, a energia principal de seu funcionamento é também a informação. Já hoje, quando nos referimos às manifestações geográficas decorrentes dos novos progressos, não é mais de meio técnico que se trata. Estamos diante da produção de algo novo, a que estamos chamando de meio técnico-científico-informacional" (SANTOS, 1996, p. 159). 
Cada elemento destacado faz parte de um conjunto de mudanças que merecem um tratamento especial de aprofundamento que não temos condições de demonstrar nesse estudo. Entretanto, é importante destacar que todas essas mudanças estão relacionadas ao desenvolvimento da reestruturação produtiva sob o regime de acumulação flexível (ANTUNES, 1999; HARVEY, 1992;), do Neoliberalismo enquanto política e da ascendência da informação enquanto elemento de conhecimento sobre os territórios.

Essas mudanças socioespaciais apontadas pelos autores foram analisadas e sistematizadas por inúmeros geógrafos que se ocuparam em investigar o desenvolvimento desigual (SMITH, 1986) do capitalismo entre os diversos países e cidades, identificando as enormes desigualdades nas formações socioespaciais. Para Santos (1996), o meio geográfico constituído após a ascendência do $\mathrm{MTCl}$, propiciou um adensamento de técnica, ciência e informação, que diferenciou as regiões e as cidades em suas formas e estruturas configurados em diferentes camadas de tempo. À camada de tempo atual um conjunto de famílias de objetos se sobrepôs ao território de maneira homogênea, possibilitando que esses objetos dessem início a um período global de difusão do conhecimento e de mobilidade das pessoas por meio de sistemas de movimento (CONTEL, 2001) cada vez mais rápidos e interligados. Tais são essas as principais características do que o autor denominou como fundamentais para as diferenciações territoriais e que também são fundamentais para o entendimento da disseminação e efeitos da COVID-19 no Brasil.

Para compreender como tais elementos possibilitaram uma maior difusão das doenças virais e em especial para compreender a dinâmica da COVID-19, particularmente no Brasil, é necessário entender as mudanças ocorridas na sociedade e no espaço geográfico após esse período, focando nas características relacionadas com a ascensão da globalização (SANTOS, 2011) e a compressão espaço-tempo (HARVEY, 1992) que fizeram com que uma nova família de objetos técnicos imprimissem maior agilidade à circulação.

No caso da COVID-19, verifica-se que os objetos geográficos influenciam tanto no aparecimento de casos, como na circulação das pessoas e objetos, que transportam o vírus. As cidades e suas características singulares vão influenciar na distribuição e na manutenção da COVID-19, por meio dos fluxos estabelecidos pelos macro-sistemas de engenharia de circulação, tais como estações ferroviárias, rodoviárias, aeroportos e portos, ou seja, as ligações pela rede urbana.

Essa constatação mostra que o vírus da COVID-19 tem maior mobilidade espacial hoje do que teria no início do século XX. Embora com diferenças significativas decorrentes da natureza do vírus e da base material à época, a pandemia da Gripe Espanhola (1918-1919) demonstrou um ritmo de disseminação mais lento que o do Coronavírus. Na cidade sul mineira de Poços de Caldas, por exemplo, a Gripe Espanhola chegou predominantemente pela via ferroviária cerca de um mês e meio após a primeira ocorrência no país, enquanto que nas cidades litorâneas do Brasil, chegou pelos portos meses após a disseminação no exterior (JUDICE, 2006).

No mundo contemporâneo, com a atual velocidade dos meios de circulação, podemos afirmar, como lembra Sposito e Guimarães (2020), que os vírus, por meio dos humanos, "saltam escalas geográficas", no sentido dado a essa expressão por Smith (1986). São, dessa maneira, transferidos de uma parcela a outra do território, atravessando continentes e oceanos, em pouco tempo, ligando pontos que são dotados de infraestruturas de circulação como grandes aeroportos e rodovias localizados mais densamente nas cidades mais conectadas, localizadas nas maiores hierarquias da rede de cidades (IBGE, 2008).

É por meio das interconexões da rede de cidades que, segundo Corrêa (2006), são direcionados os fluxos. As redes de transportes rodoviários e aeroviários, por serem aquelas prioritárias para o transporte de pessoas, ganham destaque para a análise da disseminação da COVID-19. Isso porque, geralmente, a difusão envolve tanto o contágio por vizinhança quanto a transmissão por meio da hierarquia das cidades. Sendo assim, está relacionada com as interações espaciais existentes na rede urbana brasileira e com os sistemas de transportes que as articula.

\section{Os fluxos sul mineiros}

A regionalização em mesorregiões geográficas, tomada como referência na pesquisa, foi elaborada pelo IBGE em 1989. Ela parte de critérios sociais, ambientais e da análise das redes de lugares e de comunicação (IBGE, 1990). A escolha desta regionalização em detrimento das Regiões Geográficas 
Intermediárias (IBGE, 2017) se deu em função de que aquela melhor representa a identificação regional da população.

A região de estudo, denominada pelo IBGE como mesorregião Sul e Sudoeste de Minas e popularmente chamada "Sul de Minas", abrange 146 municípios com um efetivo populacional de 2.929.424 habitantes (IBGE, 2019). Destes, apenas 4 possuem mais de 100 mil habitantes e 33 acima de 20 mil habitantes, como pode ser observado na tabela 1. Predominam os municípios com população pequena, que ora se especializam produtivamente (como no caso dos municípios de produção têxtil - Borda da Mata, Jacutinga, Monte Sião - e Extrema na produção industrial), ora são centros locais.

Tabela 1 - Maiores Municípios da Mesorregião Sul/Sudoeste de Minas Gerais

\begin{tabular}{lc}
\hline Municípios & População \\
\hline Poços de Caldas & 167.397 \\
\hline Pouso Alegre & 150.737 \\
\hline Varginha & 135.558 \\
\hline Passos & 114.679 \\
\hline Itajubá & 96.869 \\
\hline Alfenas & 79.996 \\
\hline Três Corações & 79.482 \\
\hline São Sebastião do Paraíso & 70.956 \\
\hline Três Pontas & 56.746 \\
\hline Guaxupé & 51.917 \\
\hline \multicolumn{1}{c}{ Fonte: IBGE: Estimativa Populacional 2019. }
\end{tabular}

As principais atividades econômicas da região são: agricultura cafeeira, pecuária leiteira, mineração (alumínio), indústrias (eletroeletrônicos, helicópteros, autopeças, bebidas, têxteis) e turismo (ASSOCIAÇÃO MINEIRA DE MUNICÍPIOS, 2014). Conta também 13 campi de universidades e institutos federais além de universidades particulares que, em conjunto, atraem pessoas de outras regiões do país. As cidades possuem boa qualidade de vida, sendo comum que paulistanos e cariocas se mudem para a região, especialmente após a aposentadoria.

A rede urbana da mesorregião é composta por poucas cidades médias3, que articulam a vida regional. De acordo com a Regic - Região de Influência das Cidades - 2007 (IBGE, 2008), as cidades sul mineiras se conectam principalmente com São Paulo, a exceção de Varginha e sua hinterlândia, que estabelece laços mais significativos com Belo Horizonte, como pode ser observado na tabela 2 .

Tabela 2 - Hierarquia Urbana no Sul/Sudoeste de Minas Gerais

\begin{tabular}{lcc}
\hline Município & Classificação na Hierarquia urbana & Metrópole a que se vincula \\
\hline Pouso Alegre & Capital Regional C & São Paulo \\
\hline Varginha & Capital Regional C & Belo Horizonte \\
\hline Poços de Caldas & Centro Sub-Regional A & São Paulo \\
\hline Alfenas & Centro Sub-Regional A & São Paulo \\
\hline Itajubá & Centro Sub-Regional B & São Paulo \\
\hline
\end{tabular}

\footnotetext{
3 Segundo Sposito (2006, p. 144), cidades médias podem ser entendidas como aquelas que desempenham papéis intermediários no âmbito da rede urbana.
} 
Os demais municípios são classificados como Centros de Zonas ou Centros locais, possuindo menor influência na rede de cidades sul mineiras.

A análise da Logística de Transportes 2014 (IBGE, 2014) identificou a predominância do modal rodoviário na região sul de minas, sendo composto principalmente por rodovias pavimentadas não duplicadas. A mais importante rota rodoviária da região e a Fernão Dias (BR-381), que liga São Paulo a Belo Horizonte. O único município que apresenta importância aeroviária na região é Varginha (IBGE, 2011).

Essas informações são importantes para demonstrar que os fluxos de pessoas (migração, deslocamentos semanais para trabalho e estudo, visitas a parentes, etc) e mercadorias da região Sul de Minas se dão principalmente por vias rodoviárias e para as cidades de São Paulo, Campinas, Rio de Janeiro e Belo Horizonte, grandes vetores de disseminação da COVID-19.

\section{ANÁLISE DA DISTRIBUIÇÃO DA COVID-19 NA REGIÃO SUL/SUDOESTE DE MINAS GERAIS}

A análise proposta neste artigo busca atender as demandas de informação e proteção das populações das cidades sul mineiras e oferecer um material de qualidade e confiável para gestores municipais, estaduais e população da região. Busca-se estabelecer um quadro geográfico interpretativo (GOMES, 2017) da COVID-19 no sul de Minas, a partir do cruzamento e análise geográfica de um conjunto de informações pertinentes (casos da doença, eixos rodoviários, população total e suas características demográficas). Dessa maneira, buscamos garantir que as políticas públicas voltadas ao desenvolvimento econômico e social considere a melhoria das condições de vida da população do Sul de Minas Gerais, observando as ações de inclusão, o fomento do empreendedorismo, do cooperativismo, da inovação e do direito a assistência social.

O atual estágio da pesquisa aponta para uma necessidade de se notar os eixos de circulação da COVID-19 provenientes dos estados de São Paulo e Rio de Janeiro como maiores focos de transmissão da doença para o Sul de Minas. Isso porque os dados absolutos não mostram o "movimento" do vírus, dado de suma importância para a proposição de controle e mitigação da transmissão entre a população. Somente uma análise geográfica pode revelar essa dinâmica. Como pode ser constatado no mapa 1 , a aferição dos dados promove uma exata dimensão da distribuição dos casos, porém sem constatações da expansão e tratamento da circulação do vírus.

Nesse sentido, as atualizações dos casos devem ser acompanhadas de mapas temáticos com os principais nós de contextos que ajudem em um diagnóstico preciso da doença. Um exemplo é mapa 2 que relaciona os casos absolutos de maneira proporcional em círculos com uma malha de população. Essas informações são organizadas em conjunto com os eixos de circulação de entrada no Sul de Minas provenientes de São Paulo e Rio de Janeiro pelas principais rodovias federais.

A análise do mapa 2 de casos confirmados de COVID-19 mostra que a expansão dos casos na mesorregião Sul e Sudoeste de Minas Gerais se dá seguindo as linhas de conexão dos principais eixos rodoviários. Tais eixos são aqueles provenientes das cidades com maior número de casos confirmados do Estado de São Paulo. O eixo de entrada pela Rodovia Fernão Dias, que liga São Paulo a Belo Horizonte e passa pelo sul de Minas, revela-se como o maior fluxo de dispersão para a região estudada, afetando os municípios que a margeiam, como pode ser observado no eixo de contaminação que atinge Extrema/MG, Toledo/MG e Cambuí/MG; chegando à Pouso Alegre/MG que apresenta o maior número de contaminações no Sul de Minas. Belo Horizonte, embora tenha começado posteriormente a transmissão do vírus pode ter também um papel importante na difusão do mesmo na rede urbana.

Outros eixos de contaminação possuem relevância no contexto considerado e, dentre eles, destacase o eixo proveniente do estado do Rio de Janeiro que tem seus fluxos vindos da cidade do Rio de Janeiro com 6.750 casos em 03 de Maio de 2020. A evidência é posta por conta do nível de contaminação observado tanto em cidades médias como Juiz de Fora/MG, quanto em cidades menores como Santos Dumont/MG, Belmiro Braga/MG e Matias Barbosa/MG. Ambas cidades recebem os fluxos do Rio de Janeiro por meio da rodovia BR 040, que também oferece a ligação para Varginha/MG, cidade que apresenta um número considerável de contaminações se comparada ao contexto regional abordado. Há de se destacar também os fluxos provenientes do Rio de Janeiro que chegam ao Sul de Minas por meio da Via Dutra e da BR-459, atingindo as cidades de Itajubá/MG, Pouso Alegre/MG e Poços de Caldas/MG. 


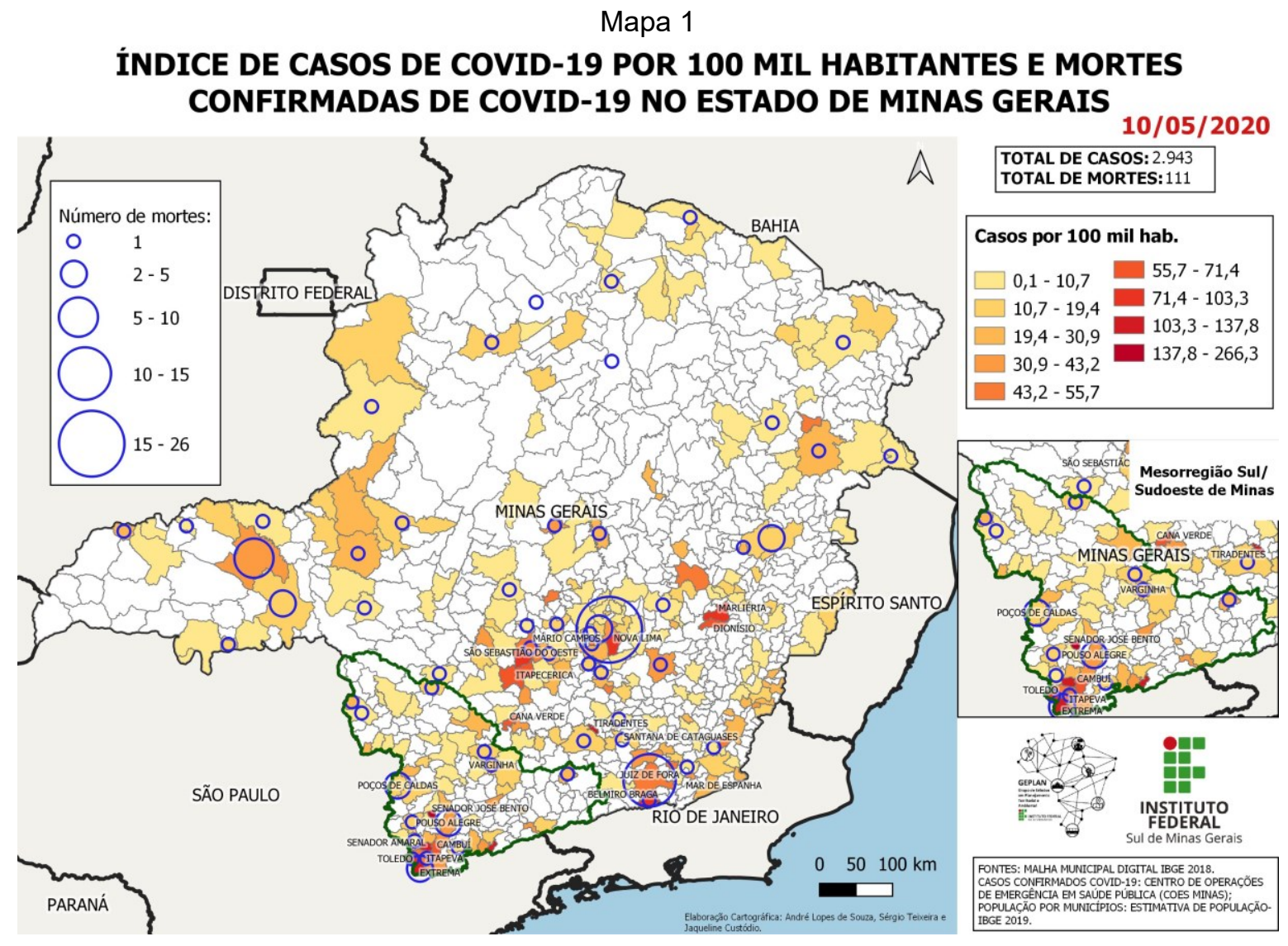

Mapa 2

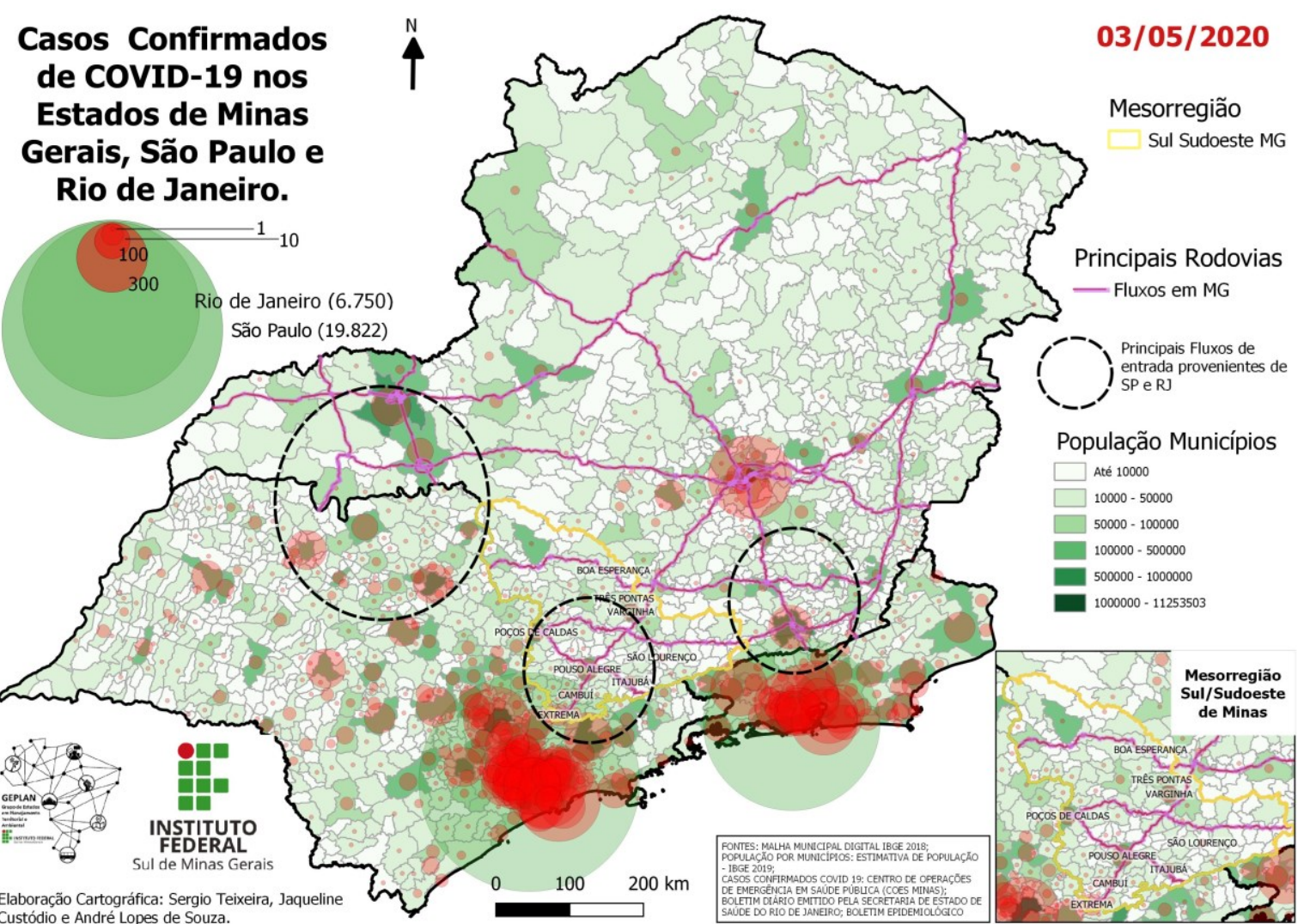


Os mapas mais recentes produzidos pela pesquisa mostram um aumento substancial nos casos e nas mortes na região Sul e Sudoeste de Minas, como pode ser visto no mapa 3.

É importante destacar que considera-se aqui a possibilidade da existência de subnotificação de dados, sobretudo nas cidades pequenas e médias interioranas. Isso porque nessas cidades há falta de equipamentos médicos de alta complexidade que realizem os testes, o que leva, em muitos casos, pessoas contaminadas não procurem o sistema de saúde quando necessário. Destaca-se que os testes são feitos somente em Belo Horizonte, cidade que lida com uma alta demanda de testes. Isso atrasa e dificulta a apreensão real do cenário da doença na região.

\section{Mapa 3}

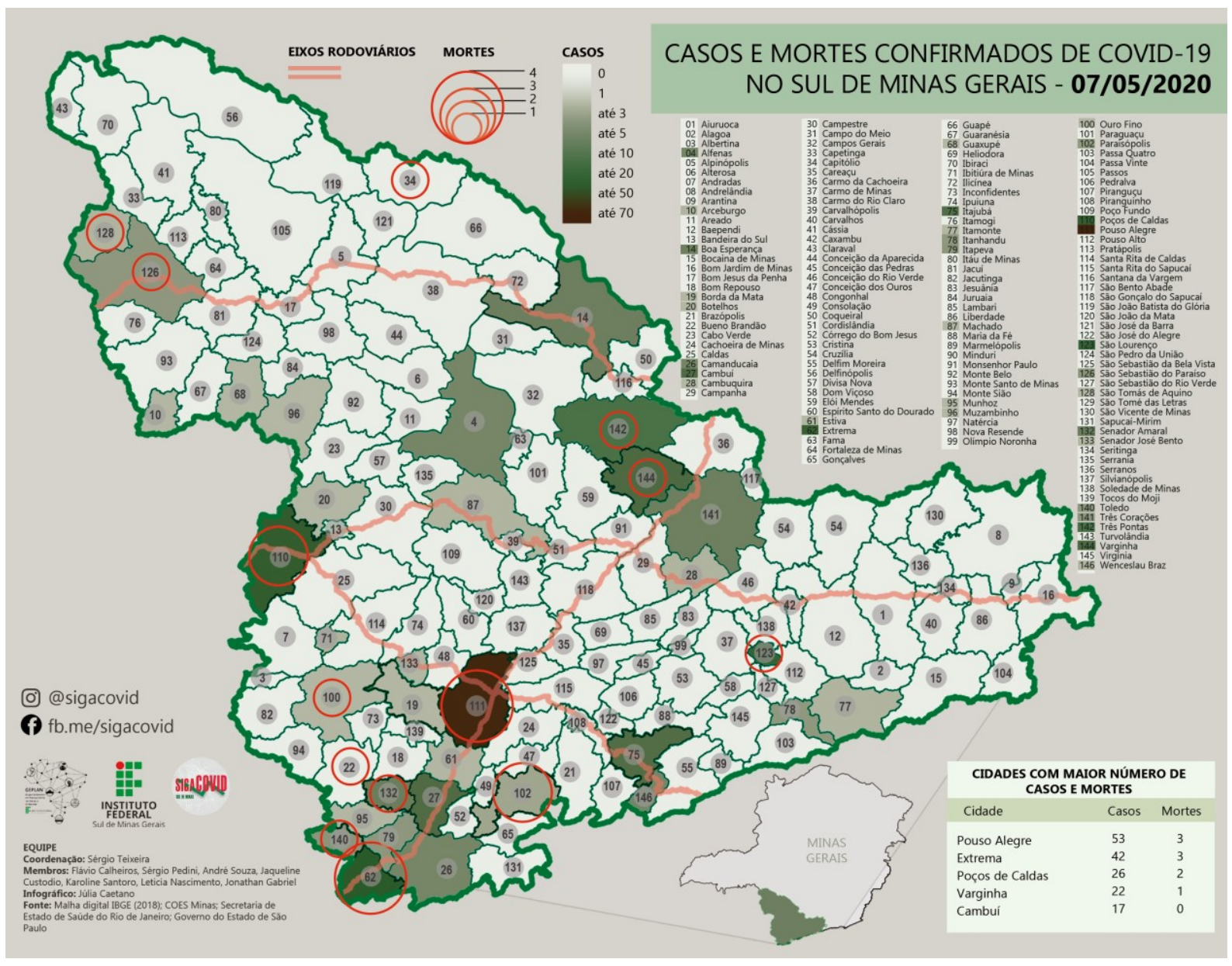

As informações discutidas até o momento devem ser analisadas em conjunto com outras características de população. Entre elas, a de maior expressão para o controle da letalidade do vírus é o acompanhamento e diagnóstico das cidades com número elevado de idosos. Tal aferição pode ser feita cruzando os dados de população municipal maior de 60 anos com o número de jovens, procedimento conhecido como índice de envelhecimento.

O mapa 4 e o mapa 5 mostram esse levantamento preliminar nas cidades do Estado de Minas Gerais com o foco na mesorregião Sul e Sudoeste de Minas. O diagnóstico inicial apresenta um mapa que relaciona casos confirmados, mortes e índice de envelhecimento. No mapa 4 pode ser analisado que as cidades do Norte de Minas são aquelas com menores índices de envelhecimento. Isso deve-se, sobretudo, às condições sociais mais severas que impactam na expectativa de vida e maior índice de mortalidade entre a população mais idosa. No eixo que se estende da região metropolitana 
de Belo Horizonte no sentido Sul de Minas o índice de envelhecimento aumenta, sendo que na região Sul, Sudoeste, Sudeste e Triangulo Mineiro estão as cidades com maiores índices do estado de Minas Gerais.

\section{CASOS E MORTES CONFIRMADAS DE COVID-19 E INDICE DE ENVELHECIMENTO DA POPULACÃO NO ESTADO DE MINAS GERAIS}

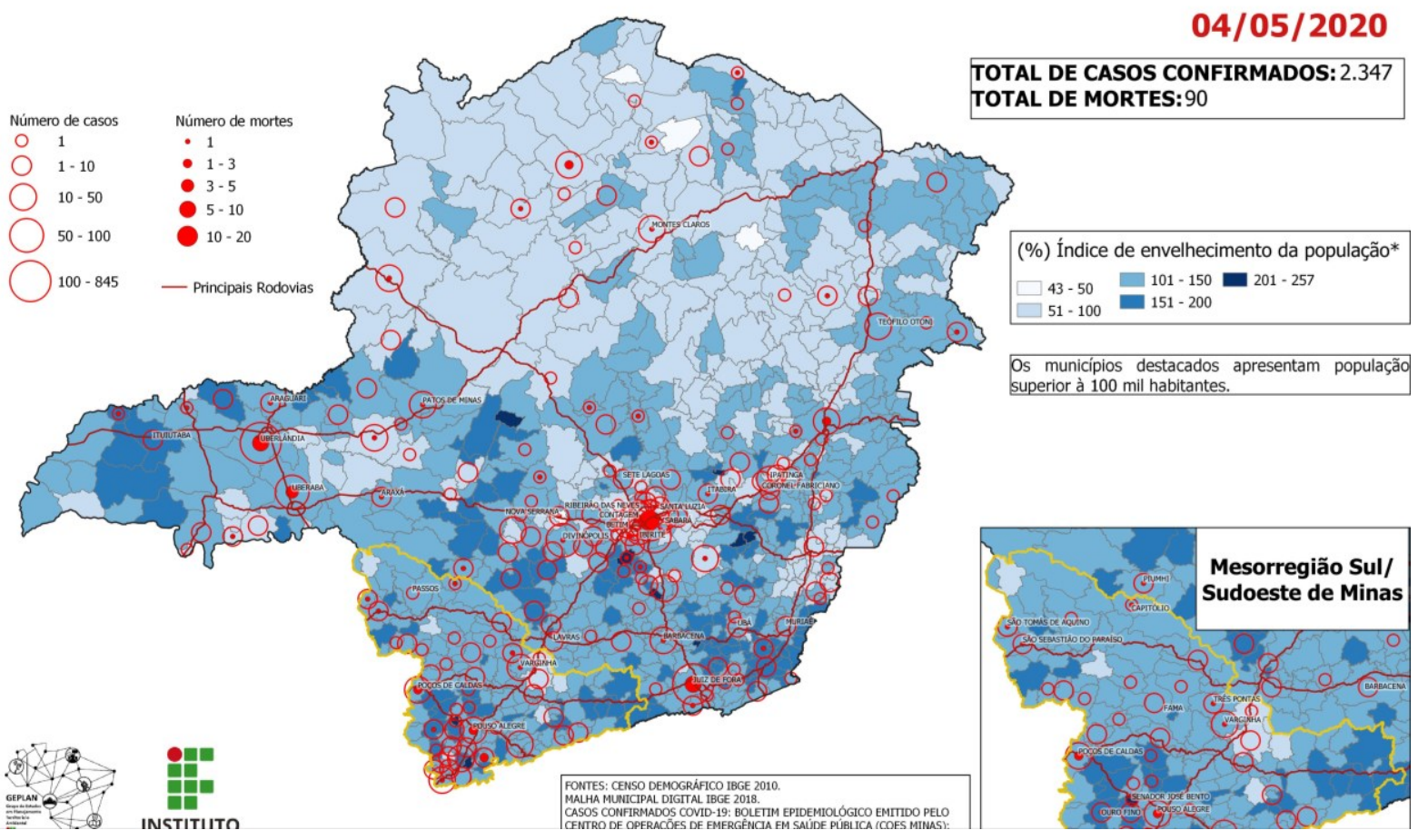

Sendo as cidades do eixo Sul de Minas as cidades com maior número de população idosa e cortadas pelos principais eixos rodoviários, devem ser dadas a elas atenção especial quanto a transmissão da COVID-19. Isso porque há uma tendência, também observada nos mapas 4 e 5, de um maior número de letalidade entre os contaminados.

\section{CONCLUSÕES E APONTAMENTOS}

A pesquisa está em estágio de análise preliminar dos dados para elaboração de hipóteses a serem posteriormente investigadas. Os próximos passos da pesquisa envolvem cruzamentos com outros dados, como disponibilidade de leitos de UTI, respiradores, eixos aéreos, entre outros. Também será agenda de pesquisa a análise qualitativa dos casos confirmados. Pretende-se chegar a um complexo sistema de informações geográficas de interesse para a análise da disseminação da COVID-19 no sul de minas.

Embora a análise dos dados ainda esteja em fase preliminar, considera-se suficientemente verificado que os eixos rodoviários e a hierarquia urbana contribuem de maneira significativa para a distribuição da COVID-19 no sul de Minas. Nesse sentido, sugere-se um encaminhamento prático por parte do poder público estadual. Deve-se dedicar especial atenção ao controle epidemiológico nas cidades de entrada dos fluxos provenientes de São Paulo (Capital e Interior) e Rio de Janeiro. Uma iniciativa seria operacionalizar postos de medição de temperatura corporal nas entradas rodoviárias dessas cidades com posterior, se necessário, encaminhamentos com níveis de prioridades para testes rápidos nesses eixos. 


\section{Mapa 5}

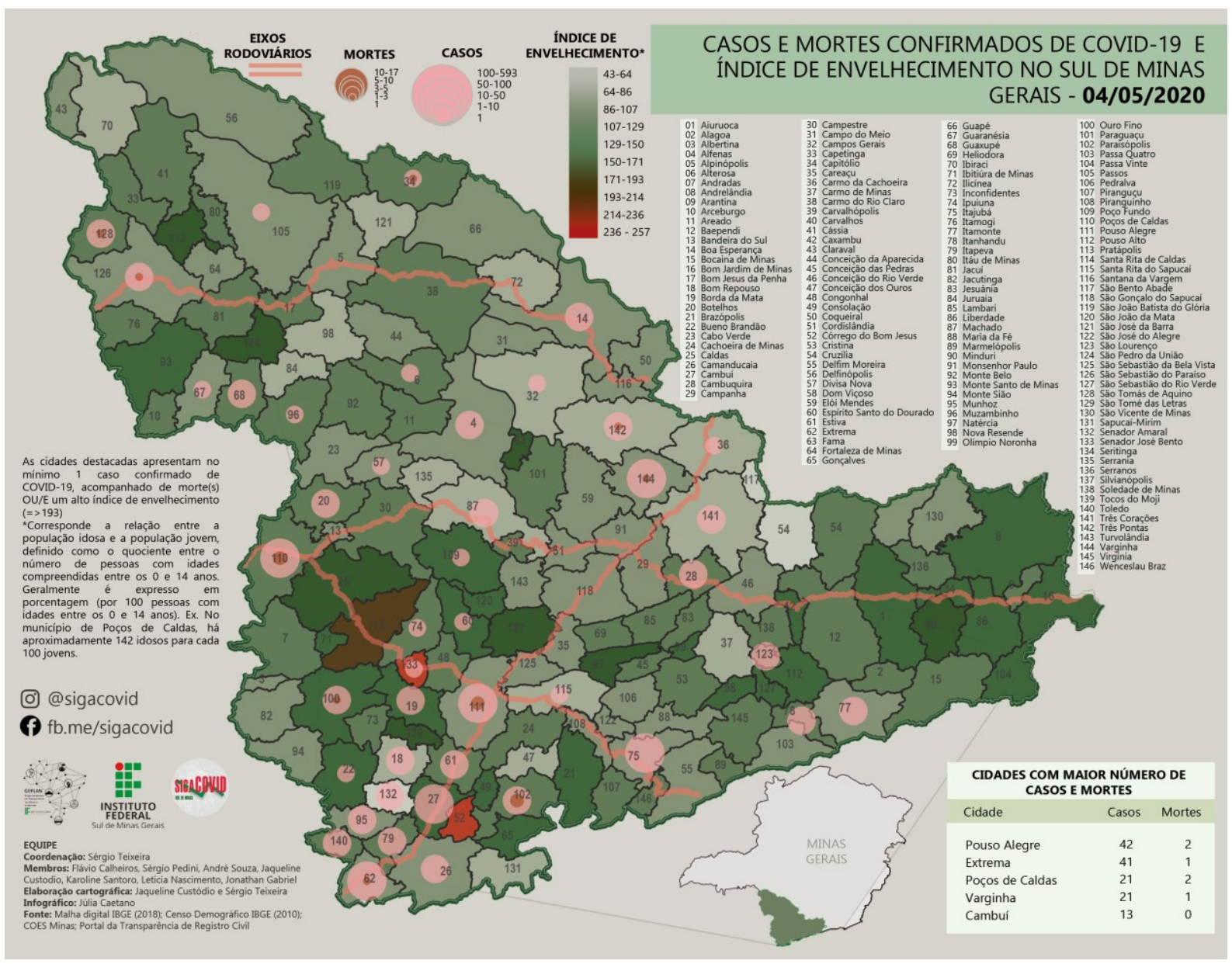

Destaca-se também que alguns dos municípios mais afetados no sul de Minas não são os de maior hierarquia na rede urbana, demonstrando a importância do contágio por vizinhança. Uma das hipóteses que a pesquisa verificará em trabalhos posteriores é que os estabelecimentos localizados às margens das rodovias de grande fluxo (postos de gasolina, conveniência, restaurantes, borracharias, etc) podem ser vetores de transmissão da COVID-19. Isso explicaria por que municípios como Extrema (36 mil habitantes) e Cambuí (30 mil) têm alto número proporcional de ocorrências. Ambos municípios se localizam no principal eixo rodoviário, o da Fernão Dias, embora não sejam as cidades posicionadas nas mais altas hierarquias na rede urbana sul mineira.

Por fim, a pesquisa demonstrou que a análise geográfica contribui para o entendimento, em diversas escalas geográficas, da disseminação da COVID-19 e para o seu enfrentamento. Na escala local das cidades, indicamos que é preciso gerir os fluxos de pessoas; na escala mesorregional, identificamos que as rodovias são os principais eixos de disseminação da doença; e na escala estadual, discutimos que alguns municípios podem apresentar maior vulnerabilidade aos efeitos da doença em virtude da maior proporção de idosos.

As orientações aos poderes públicos continuam sendo a não flexibilização do isolamento social, maior atenção à população idosa e instalação de pontos de controle de medição de temperatura e testes rápidos nos eixos que ligam as principais cidades do Sul e Sudoeste de Minas aos estados de São Paulo e Rio de Janeiro.

Municípios como Extrema/MG, Córrego de Bom Jesus/MG, Senador José Bento/MG, Ouro Fino/MG e Poços de Caldas/MG revelam-se mais sensíveis à letalidade do vírus, uma vez que apresentam alto 
índice de envelhecimento e têm ligações diretas ou subsidiárias com grandes eixos de ligações rodoviárias.

\section{REFERÊNCIAS}

ANTUNES, Ricardo. Os sentidos do Trabalho: ensaio sobre a afirmação e a negação do trabalho. São Paulo: Boitempo, 1999.

ASSOCIAÇÃO MINEIRA DE MUNICÍPIOS. Caracterização econômica das regiões de planejamento. 2014. Disponível em: https://portalamm.org.br/caracterizacao-economica-das-regioesde-planejamento/

CATÃO, R. C. Expansão e consolidação do complexo patogênico do dengue no Estado de São Paulo : difusão espacial e barreiras geográficas. $257 \mathrm{f}$. Tese (Doutorado) - Universidade Estadual Paulista "Júlio de Mesquita Filho" Faculdade de Ciências e Tecnologia, 2011.

CONTEL. F. B. Os sistemas de movimento do território brasileiro. In: SANTOS \& SILVEIRA. O Brasil, EDUSP. 2001.

CORREAA, R. L. Estudos sobre a Rede Urbana. Rio de Janeiro: Bertrand-Brasil, 2006.

GLUBER, Duane. Dengue and Dengue Hemorrhagic Fever. Clinical Microbiology Reviews, Philadelphia, v. 3, n. 11, p. 480-496, jul., 1998. https://doi.org/10.1128/CMR.11.3.480

GOMES, P.C.C. Quadros geográficos: uma forma de ver, uma forma de pensar. Bertrand Brasil: Rio de Janeiro. 2017.

HARVEY, D. A condição pós-moderna. São Paulo: Loyola, 1992.

IBGE. Divisão regional do Brasil em mesorregiões e microrregiões geográficas. Rio de Janeiro: IBGE, 1990.

Regiões de Influência de Cidades (REGIC). Rio de Janeiro: IBGE, 2008.

Logística de Transportes 2014. Rio de Janeiro: IBGE, 2014.

Ligações aéreas 2010. Estudo, Rio de Janeiro: IBGE, 2011.

Divisão regional do Brasil em regiões geográficas imediatas e regiões geográficas intermediárias: 2017. Rio de Janeiro: IBGE, 2017.

Estimativas da população residente para os municípios e para as unidades da federação $\overline{c o m}$ data de referência em $1^{\circ}$ de julho de 2019: [notas metodológicas]. Rio de Janeiro, 2019.

JUDICE, L.R. A gripe espanhola em Poços de Caldas -1918. Poços de Caldas: Sul Minas, 2006.

MARINI, R. M. Dialéctica de la Dependencia. 3. ed. México: Série Popular Era, 1977.

SANTOS, M. A Natureza do Espaço: espaço e tempo, razão e emoção. 3. ed. São Paulo: Hucitec, 1996.

Técnica Espaço Tempo: globalização e meio técnico-científico informacional. $3^{a}$ ed. São Paulo: HUCITEC, 1997.

O espaço dividido: os dois circuitos da economia urbana dos países subdesenvolvidos. São Paulo: Editora da Universidade de São Paulo, [1979] 2004.

A urbanização brasileira. São Paulo: EDUSP, [1993] 2005.

Por uma outra globalização. Rio de Janeiro/São Paulo: Editora Record, 2011.

SMITH, N. Desenvolvimento Desigual. Rio de Janeiro: Bertrand Brasil, 1986.

SPOSITO, M. B. E. O desafio metodológico da abordagem interescalar no estudo de cidades médias no mundo contemporâneo. Cidades, V. 3, N. 5, p. 143-157, 2006.

SPOSITO, M. E. B.; GUIMARÃES, R. B. Por que a circulação de pessoas tem peso na difusão da pandemia. Em: https://www2.unesp.br/portal\#!/noticia/35626/por-que-a-circulacao-de-pessoas-tempeso-na-difusao-da-pandemia, 2020. 